\title{
Correction to: Superoxide dismutase activity as a predictor of adverse outcomes in patients with nonischemic dilated cardiomyopathy
}

\author{
Ewa Romuk ${ }^{1}$ - Wojciech Jacheć ${ }^{2}$ - Ewa Kozielska-Nowalany ${ }^{2} \cdot$ Ewa Birkner $^{1}$ - Aleksandra Zemła-Woszek ${ }^{1}$. \\ Celina Wojciechowska ${ }^{2}$
}

Published online: 18 June 2019

(C) The Author(s) 2019

\section{Correction to: Cell Stress and Chaperones https://doi.org/10.1007/s12192-019-00991-3}

Due to an unfortunate turn of events, part of the data in the columns HR, 95\% CI, and $p$ is missing from Figs. 4, 5, 6, 7, 8, and 9 of the original publication. The correct figures and their captions are published here and should be treated as definitive.

The online version of the original article can be found at https://doi.org/ 10.1007/s12192-019-00991-3

Ewa Romuk

eromuk@gmail.com

1 Department of Biochemistry, School of Medicine with the Division of Dentistry, Medical University of Silesia, Jordana 19 Street, 41-808 Zabrze, Poland

2 Second Department of Cardiology, School of Medicine with the Division of Dentistry, Medical University of Silesia, M. C.

Skłodowskiej 10 Street, 41-808 Zabrze, Poland 


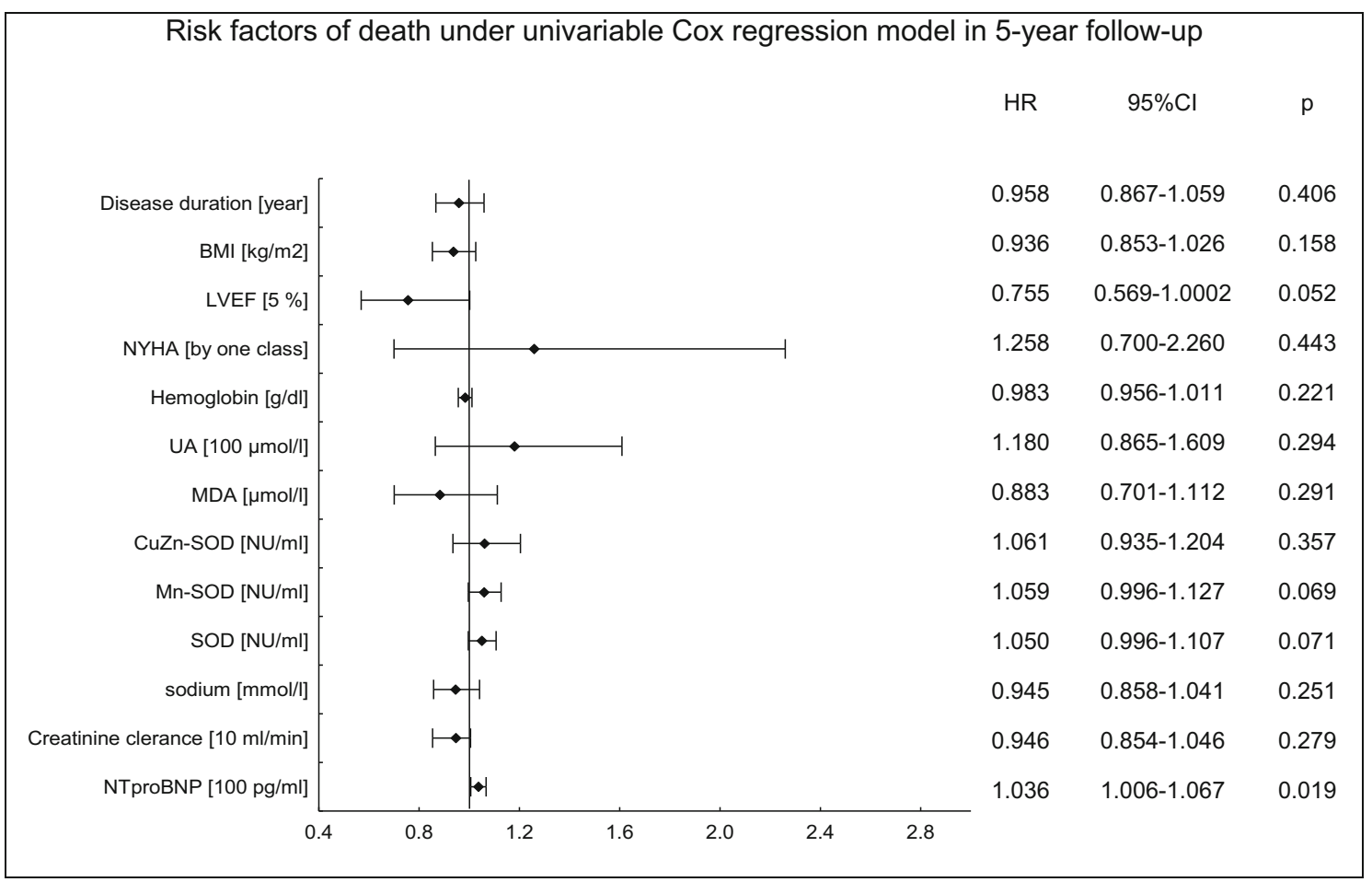

Fig. 4 Cox proportional-hazards regression for 5-year mortality rate in relation to biochemical, demographical, and clinical factors

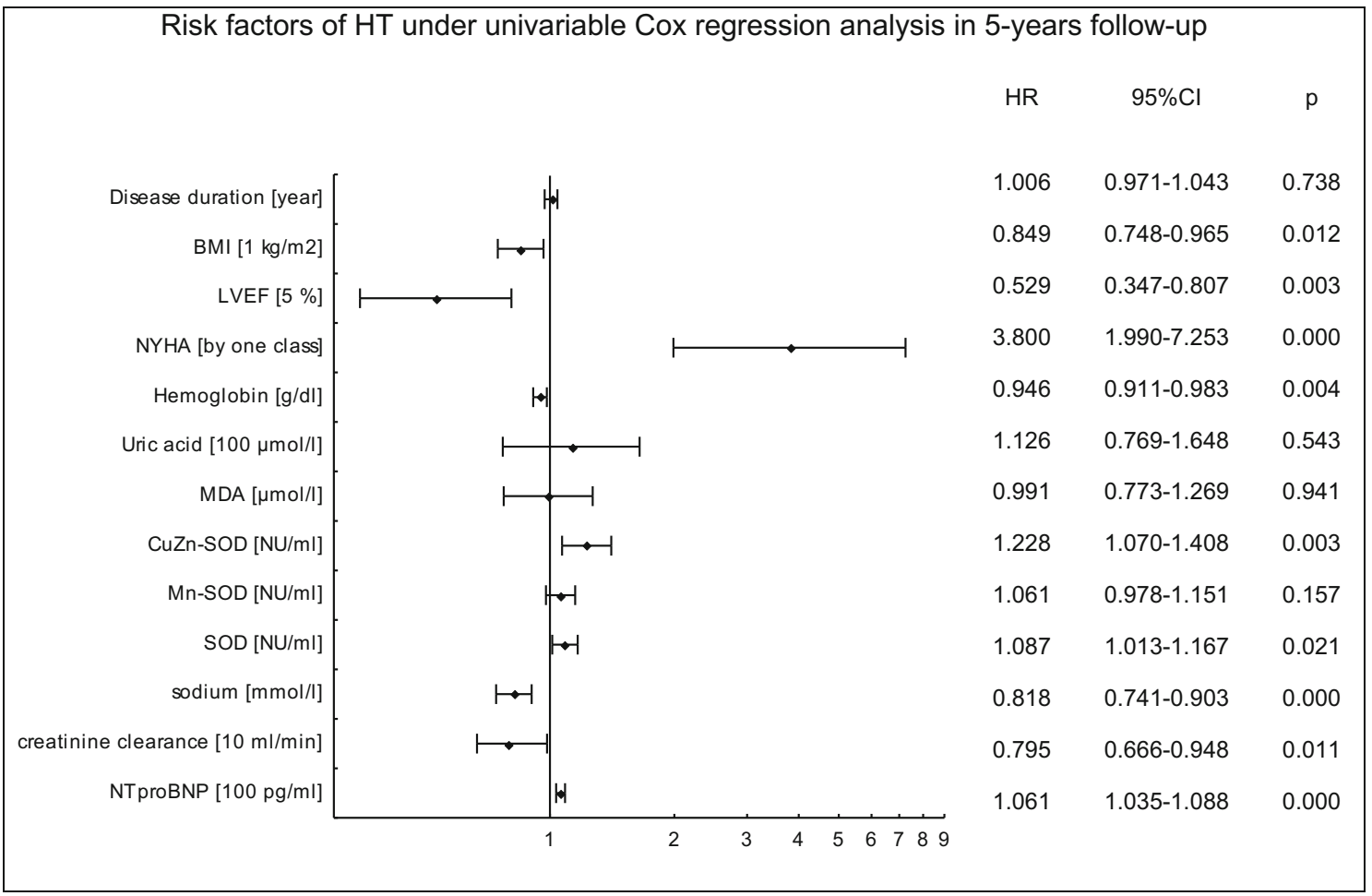

Fig. 5 Cox proportional-hazards regression for HT rate in relation to biochemical, demographical, and clinical factors 


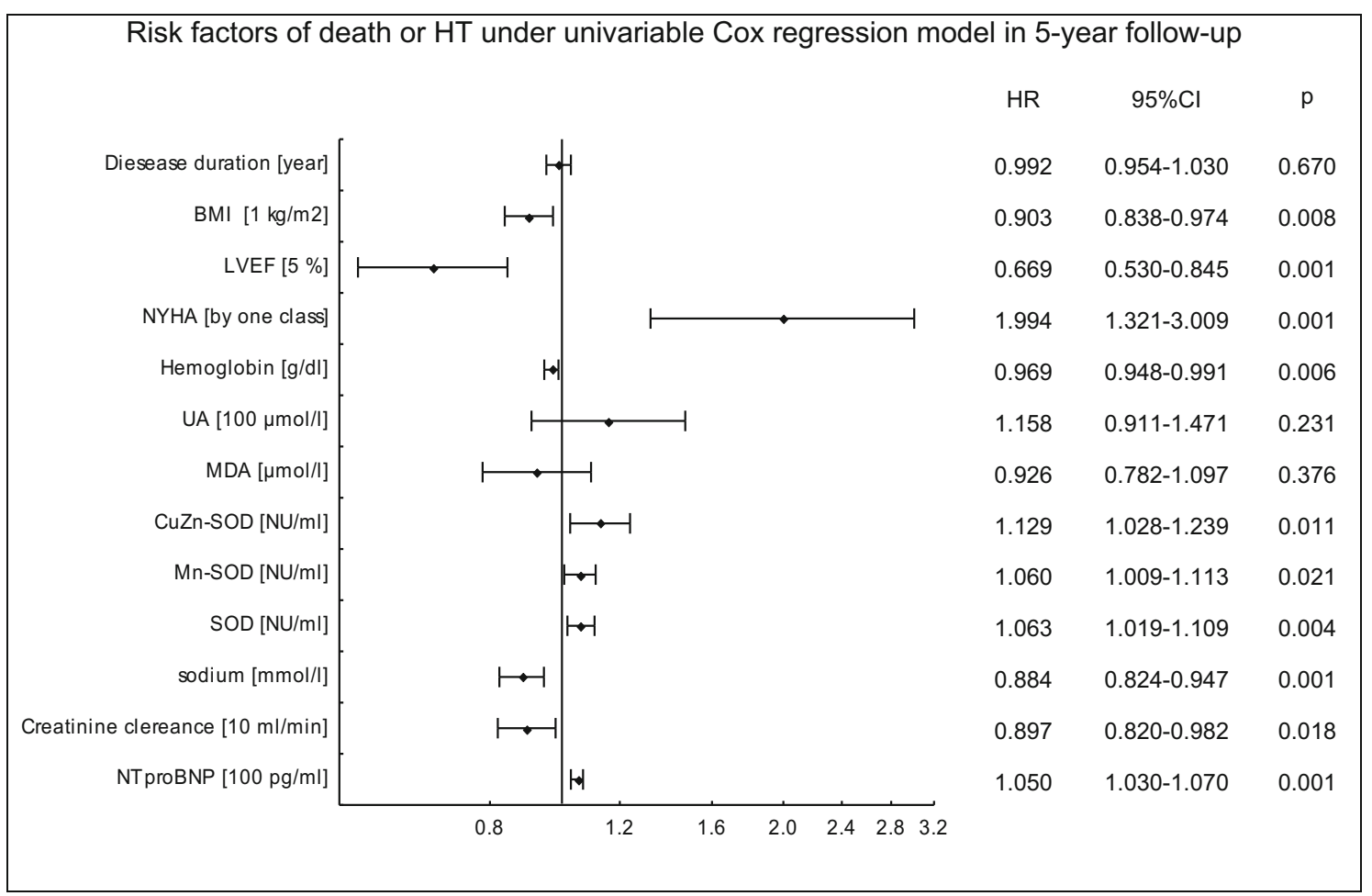

Fig. 6 Cox proportional-hazards regression for 5-year mortality or OHT rate in relation to biochemical, demographical, and clinical factors

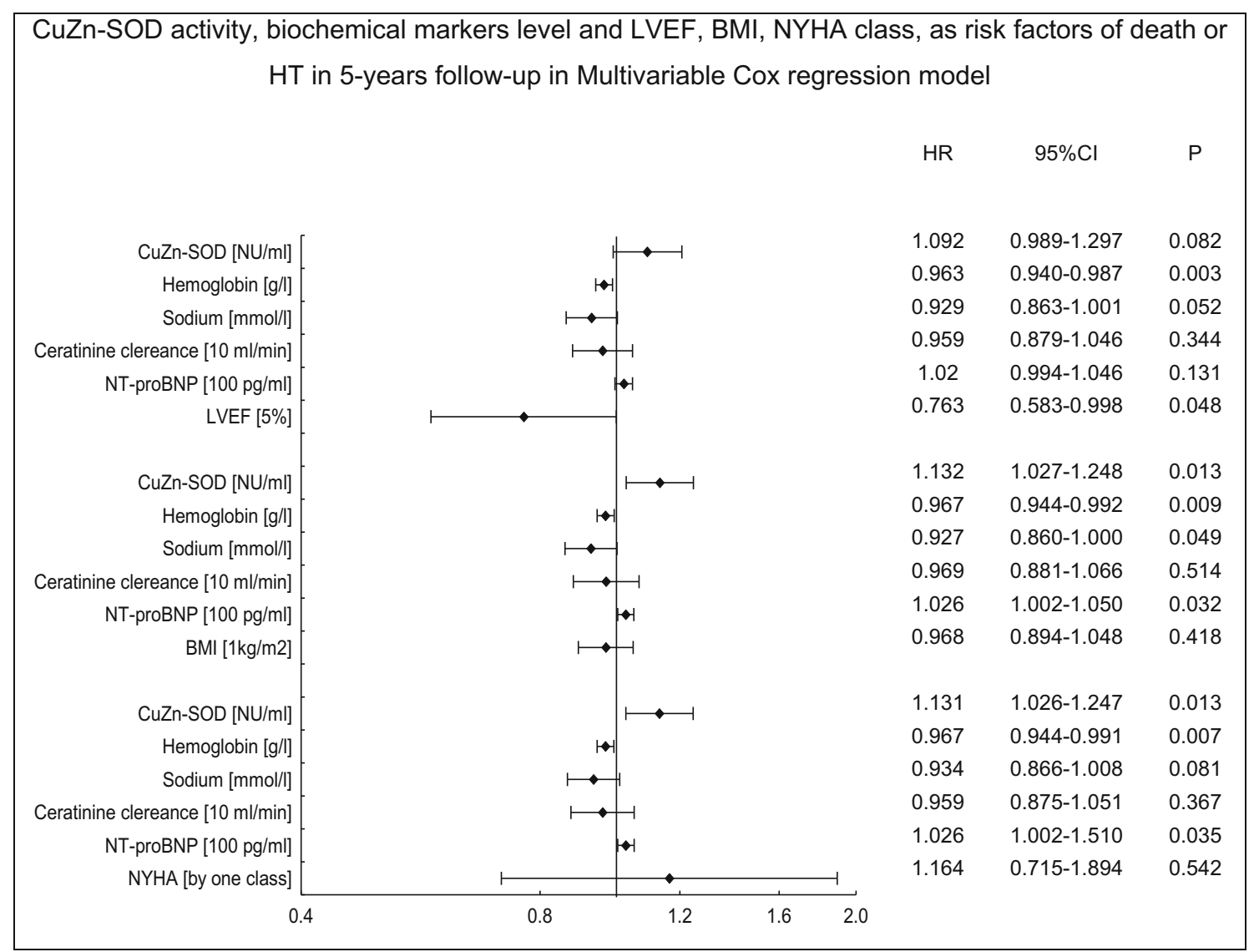

Fig. 7 Multivariable Cox proportional-hazards regression for CuZnSOD activity, LVEF, BMI, NYHA class, and biochemical parameters as risk factors of death or HT in 5-year follow-up 


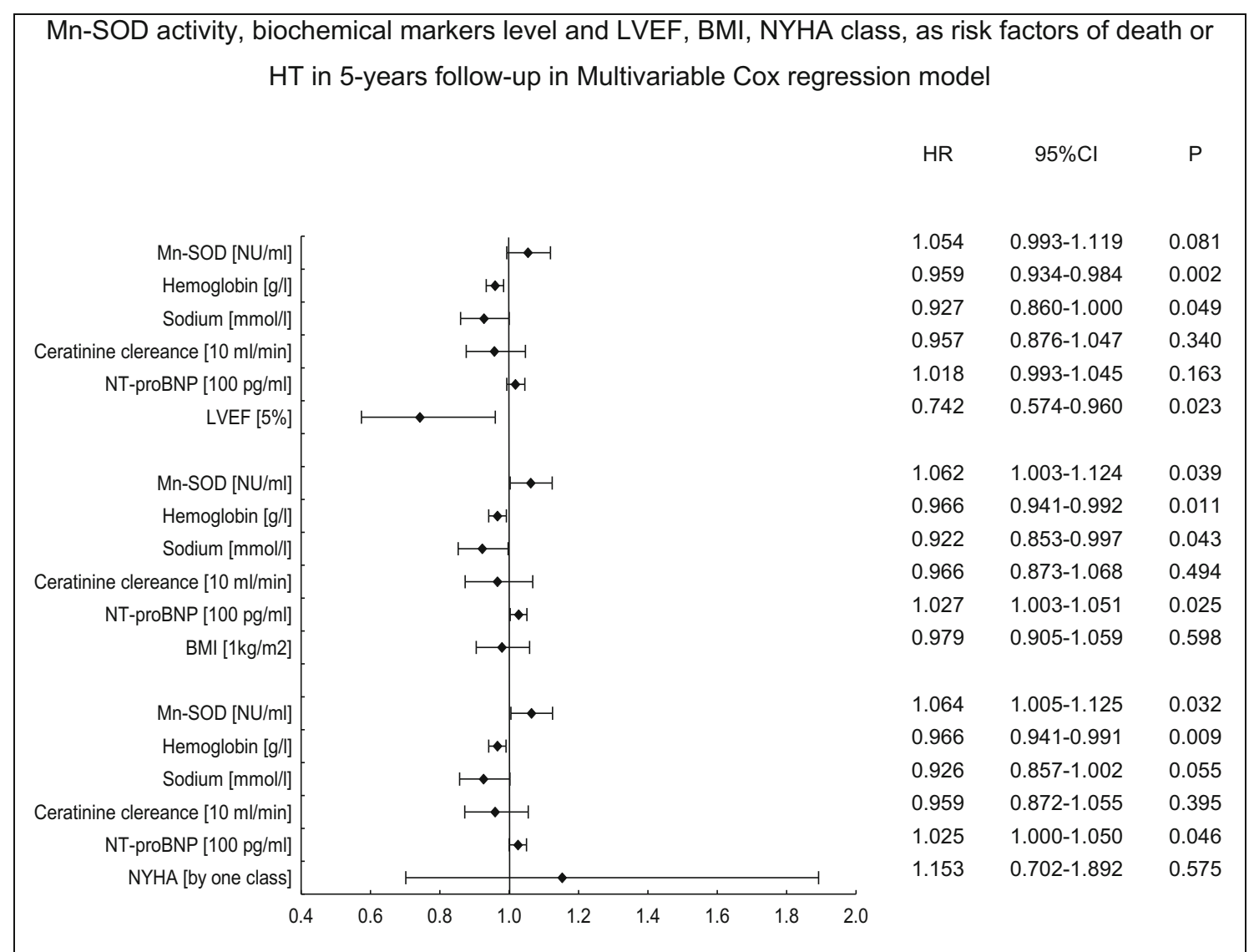

Fig. 8 Multivariable Cox proportional-hazards regression for MnSOD activity, LVEF, BMI, NYHA class, and biochemical parameters as risk factors of death or HT in 5-year follow-up 


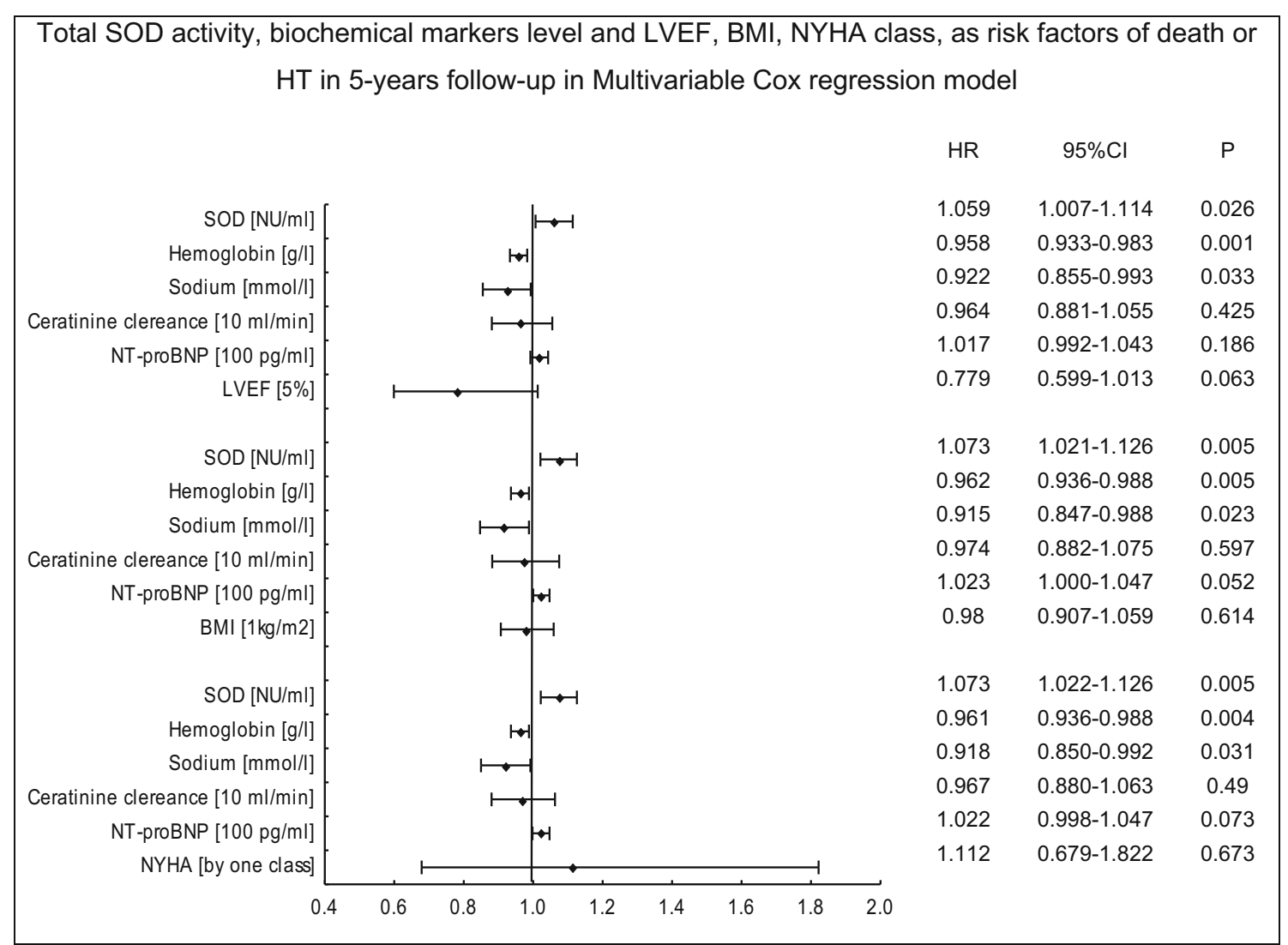

Fig. 9 Multivariable Cox proportional-hazards regression for total SOD activity, LVEF, BMI, NYHA class, and biochemical parameters as risk factors of death or HT in 5-year follow-up

Publisher's note Springer Nature remains neutral with regard to jurisdictional claims in published maps and institutional affiliations. 